\section{Intravitreal bevacizumab (Avastin) for primary treatment of diabetic macular oedema}

A Özkiriş

Keywords: intravitreal bevacizumab; diabetic macular oedema; primary treatment.

\section{Introduction}

Macular oedema is the most common cause of visual impairment in diabetic patients. The exact pathogenesis of diabetic macular oedema (DME) has not been elucidated, although a breakdown of the inner blood-retinal barrier seems to be a reasonable explanation. The important pathophysiology of DME is the loss of retinal capillary pericytes, resulting in increased vascular permeability. ${ }^{1-6}$ The 3 -year risk of moderate visual loss due to macular oedema was $32 \%$ in the Early Treatment Diabetic Retinopathy Study (ETDRS). Focal macular laser photocoagulation has been shown to be effective in the treatment of DME in a large prospective multicenter randomized clinical trial of ETDRS. ${ }^{7}$ However, some treated eyes may be resistant to laser photocoagulation or efficient laser treatment could not be performed due to diffuse macular oedema. Therefore, the failure of laser photocoagulation in these eyes has prompted interest in other treatment modalities, such as intravitreal triamcinolone acetonide (IVTA) injection, ${ }^{4,5,8,9}$ pars plana vitrectomy, ${ }^{10,11}$ or treatment with protein kinase C inhibitors. ${ }^{12}$

Retinal hypoxia is the primary cause of diabetic retinopathy, which increases expression of vascular endothelial growth factor (VEGF). As known, VEGF is a potent inducer of vascular permeability that has been shown to cause leakage from retinal vessels and contribute to DME. ${ }^{13}$ Bevacizumab (Avastin, Genentech Inc., South San Francisco, CA, USA), a full length, humanized monoclonal antibody against VEGF,
The authors have no financial or proprietary interest in any products used in this study.
Received: 11 June 2007 Accepted in revised form: 10 November 2007 Published online: 25 April 2008 \begin{abstract}
clinical course of macular oedema, and may therefore be a promising approach in the primary treatment of diabetic macular oedema. Eye (2009) 23, 616-620; doi:10.1038/eye.2008.40; published online 25 April 2008

observed.

Conclusion Intravitreal bevacizumab application provides significant improvement in visual acuity of diabetic patients and
\end{abstract}


also binds and inhibits all the biologically active forms of VEGF, and approved by the Food and Drug

Administration for the treatment of metastatic colorectal cancer. ${ }^{14,15}$ Bevacizumab has been used for intravitreal injection in patients with choroidal neovascularization, iris neovascularization, vitreous haemorrhage, and macular oedema, and shown to provide beneficial effects in such patients. ${ }^{16-20}$ However, there is only one study showing the beneficial effect of intravitreal bevacizumab therapy for persistent diffuse DME. ${ }^{20}$ Therefore, this study investigated the efficacy of intravitreal bevacizumab for primary treatment of DME by using Heidelberg retinal tomograph II (HRT II).

\section{Patients and methods}

A total of 30 eyes of 30 diabetic patients who underwent intravitreal bevacizumab injection as the primary treatment for DME were included in this study. There were 13 women and 17 men, and the mean age of the patients was $56.1 \pm 8.5$ years (range, $40-73$ years). All patients had macular oedema with hyperfluorescent leakage on fundus fluorescein angiography. The mean duration of DME was 7.4 \pm 3.9 months (range, 3-16 months). A detailed history of medication was obtained, and the patients were excluded from the study if they had uncontrolled systemic hypertension, thromboembolic events, severe renal dysfunction, nephrotic syndrome, and dysproteinemias or receiving vasoactive drugs or antioxidant.

Before intravitreal bevacizumab injection, some eyes had received peripheral scatter laser photocoagulation to ablate ischaemic areas or neovascularization, but no eyes received any treatment for DME. Intravitreal injection of bevacizumab $(2.5 \mathrm{mg} / 0.1 \mathrm{ml})$ was offered as the first treatment of DME. All patients were fully informed about the experimental character of the treatment and informed consent was obtained from each patient. The study followed the tenets of Declaration of Helsinki. Baseline parameters were documented including bestcorrected visual acuity (BCVA), oedema map values of HRT II, fundus fluorescein angiography (FFA) findings, and IOP. BCVA for each eye was ascertained using Snellen chart, which was situated $20 \mathrm{ft}$ (approximately $6 \mathrm{~m}$ ) away from the patient before intravitreal applications, and then all eyes were tested with the same correction throughout the follow-up period. The proper average VA was computed by converting the value to the LogMAR equivalent, and taking the average of the LogMAR values as described by Holladay. ${ }^{21}$ Statistical calculations were performed using LogMAR values for VA. All intravitreal injections were performed by the same surgeon $(\mathrm{AO})$ under topical anaesthesia. The drug was drawn under sterile conditions from a bevacizumab infusion bottle. The lid was prepared with povidoneiodine 5\% applied directly to the eye, and bevacizumab was injected into the anterior vitreous $3.5 \mathrm{~mm}$ posterior to the limbus in pseudophakic eyes and $4.0 \mathrm{~mm}$ posterior to the limbus in phakic eyes with a tuberculin syringe and 27-gauge needle. A cotton-tipped applicator was applied at the injection site immediately after the removal of the needle to prevent reflux. Indirect ophthalmoscopy was used to confirm the central retinal artery perfusion. Topical ciprofloxacin drops were applied four times daily for 1 week.

The eyes were examined after 1 week and every 4 weeks. Response to the treatment was monitored by VA assessment, FFA, and HRT II. Potential drug- or injection-related complications were also recorded, if present. Patients received reinjections when there was a recurrence of DME, and the recurrence was considered when there was a decrease in BCVA associated with an increase of intraretinal fluid due to macular oedema on FFA and/or HRT II.

The analysis of macular oedema was performed using the HRT II Macular oedema module. Oedema map value of HRT II was used to evaluate the changes at the macula after bevacizumab injection. The HRT II is a confocal laser scanning system that requires a series of optical section images at different locations of the focal plane. Technical details of the instrument have been described elsewhere. ${ }^{22}$ The patient's macula was focused on the monitor screen by adjusting the location of the focal plane, the best focus being directly related to subject's refractive error. The 670-nm wavelength diode laser was used to image the macula using a $15^{\circ}$ by $15^{\circ}$ field of view. The total scan depth was adjusted according to the thickness of the structure to be analysed from 0.50 to $4.00 \mathrm{~mm}$.

\section{Statistical analysis}

Results are presented as means \pm SD. Statistical evaluation of the data was performed with two-way ANOVA. The difference was considered statistically significant when $P$-value was lower than 0.05 .

\section{Results}

All patients had clinically significant macular oedema according to the ETDRS classification at the baseline examination and completed 12 weeks of follow-up. Eighteen patients (60\%) had PDR, and all these patients had had prior scatter photocoagulation at least 6 months before injection. Seventeen cases received a second (56.6\%) intravitreal injection of bevacizumab, and two $(6.6 \%)$ needed a third injection. The mean VAs, oedema map values, and intraocular pressures (IOPs) of the 
Table 1 Mean LogMAR value for the visual acuities, EM values, and IOPs of patients before and after intravitreal bevacizumab injection

\begin{tabular}{lccc}
\hline & LogMAR value & EM value & IOPs $(\mathrm{mmHg})$ \\
\hline PI & $1.09 \pm 0.23$ & $2.4 \pm 0.6$ & $15.0 \pm 2.3$ \\
1 month & $0.90 \pm 0.17$ & $1.7 \pm 0.5$ & $14.8 \pm 2.5$ \\
3 months & $0.81 \pm 0.24$ & $1.7 \pm 0.4$ & $15.1 \pm 2.4$ \\
LV & $0.77 \pm 0.26$ & $1.6 \pm 0.4$ & $14.9 \pm 2.5$ \\
\hline
\end{tabular}

$\mathrm{EM}=$ Oedema $\quad$ map; $\quad \mathrm{IOP}=$ intraocular $\quad$ pressure; $\mathrm{LV}=$ last $\quad$ visit $\mathrm{PI}=$ preinjection

patients before and after intravitreal bevacizumab injection are presented in Table 1 . There were statistically significant differences in VA after bevacizumab injection when compared with pretreatment values (for each, $P<0.001)$. After a mean follow-up period of 5.6 months, VA increased in 24 of 30 eyes with a mean of $2.4 \pm 1.6$, $2.7 \pm 1.9$ and $2.8 \pm 2.0$ Snellen lines at the 1-, 3-month, and last visit follow-up intervals, respectively. VA remained unchanged in five eyes, and decreased in one eye and showed increased fluorescein leakage on FFA.

Twenty-seven eyes showed a reduction in macular oedema map value after intravitreal bevacizumab injection. Mean oedema map value decreased from a baseline value of $2.4 \pm 0.6$ to a value of $1.6 \pm 0.4$ at the last visit control examinations. Mean reduction of oedema map values at 1-, 3-month, and last visit was 29.1, 29.1 , and $33.3 \%$, respectively and the differences were statistically significant when compared with preinjection values (for each, $P<0.001$ ).

Average IOP values at 1-, 3-month, and last visit were not statistically significant when compared with preinjection values. Mild anterior chamber cellular reaction was observed in four eyes (13.3\%), but the inflammation resolved within a week with topical corticosteroid. No other injection- or drug-related complications were observed.

\section{Discussion}

DME is the most important cause of VA impairment in patients with diabetes mellitus and may be localized or diffused. The prognosis of diffused macular oedema is poorer when compared with focal oedema. Although the exact pathophysiologic mechanism responsible for DME remain uncertain, the disruption of the inner bloodretinal barrier is known to be associated with metabolic alterations affecting the retinal epithelium or retinal vascular endothelium. ${ }^{1-4}$ The ETDRS ${ }^{7}$ demonstrated the beneficial effect of laser photocoagulation on preventing visual loss in eyes with diffuse DME. However, macular oedema may persist in some eyes despite laser treatment. IVTA injection appears to be a promising treatment for DME, the treatment is not without risks, and complications can be attributed to the injection procedure or to the corticosteroid suspension. Reported injection-related complications include endophthalmitis, lens injuries, vitreous haemorrhage, and retinal detachment. ${ }^{23,24}$ Corticosteroid-associated adverse events include IOP elevation, cataract progression, pseudoendophthalmitis, and pseudohypopyon. Moreover, the efficacy of IVTA is transient and repeated injections are required.

VEGF plays an important role in breakdown of the blood-retina barrier with increased vascular permeability resulting in retinal oedema. Therefore, anti-VEGF therapy may be a promising treatment option for ocular neovascularization and DME. Intravitreal injection of pegaptanib (anti-VEGF aptamer) has recently demonstrated promising results for DME. Cunningham et $a l^{25}$ reported that patients who had underwent intravitreal injection of pegaptanib had better VA outcomes with reduction in central retinal thickness and less additional therapy with laser photocoagulation. More recently, intravitreal bevacizumab has been used to reduce the breakdown of the inner blood-retinal barrier, extravasation from leaking blood vessels, and inhibition of neovascularization. They inhibit the release of VEGF, contribute to the integrity of the inner blood-retinal barrier, reduce extravasation from leaking blood vessels, and have beneficial effect in the prevention and treatment of macular oedema. ${ }^{20,26,27}$ The safety of intravitreal bevacizumab has been confirmed by previous animal studies and human trials, ${ }^{28,29}$ and intravitreal injection of bevacizumab has recently been reported to be effective in macular oedema of various etiologies..$^{20,30-33}$

Results of our study suggest that intravitreal bevacizumab injection appears to be effective in the primary treatment of DME. In our study, 24 eyes showed an improvement in VA with a decrease in fluorescein leakage on FFA (Figure 1a and $b$ ). Oedema map values of HRT II showed a reduction in 27 eyes (Figure 2a and b). The results of our study confirm previous reports showing the beneficial effect of intravitreal bevacizumab in the treatment of DME. In a recent study with an intravitreal injection of $1.25 \mathrm{mg}$ bevacizumab, Haritoglou et $a l^{20}$ reported an improvement in VA from a baseline value of $0.86 \log$ MAR to a value of $0.75 \log$ MAR after 6 weeks of injection in patients with DME who did not respond to other treatments such as photocoagulation, IVTA injection, or vitrectomy. An increase in VA of at least three lines was observed in 15 of 51 eyes at a 6-week follow-up, and in 6 of 23 eyes completing 12 weeks of follow-up. Mean reduction in central macular thickness was $16.9 \%$ at 6 weeks, $24.75 \%$ at 12 weeks after the injection. In this study, VA increased with a mean of 2.4, 2.7, and 2.8 Snellen lines at 1-, 3-month, and last visit follow-up intervals, respectively. This high success in our 

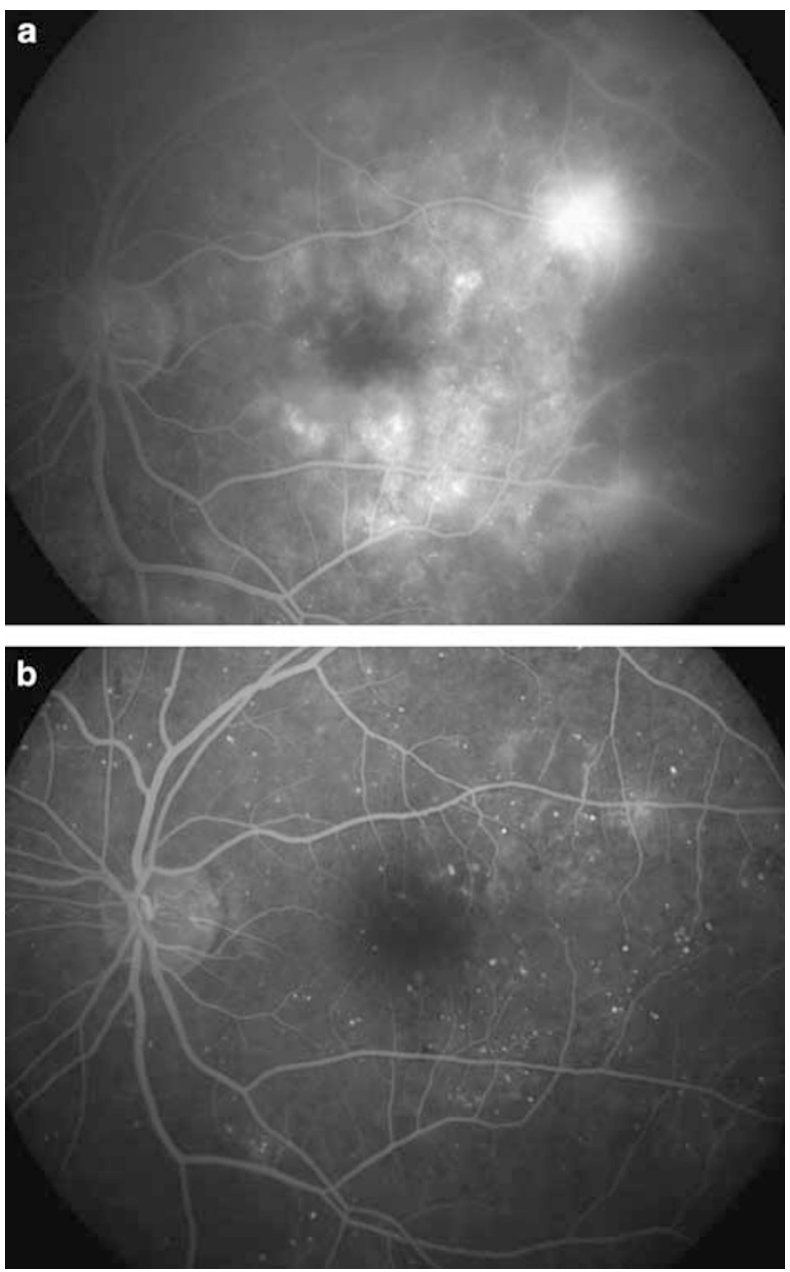

Figure 1 Late phase of FFA of a patient. (a) Before injection (b) 6 weeks after intravitreal bevacizumab injection. Note the decrease in fluorescein leakage after treatment.

study may be explained by performing intravitreal bevacizumab injection as the primary treatment of DME or a short duration of DME in our patients or using of $2.5 \mathrm{mg}$ of bevacizumab.

After intravitreal bevacizumab injection, complications such as anterior chamber inflammation, endophthalmitis, vitreous haemorrhage, subconjunctival haemorrhage, and retinal detachment may occur. In our study, four eyes developed mild anterior segment inflammation, which completely resolved in a week after topical application of corticosteroids. No other complications were observed during the follow-up period.

This study has some differences from previous studies. First, the dosage of bevacizumab injected as the primary treatment of macular oedema. Haritoglou et $a l^{20}$ used $1.25 \mathrm{mg}$ bevacizumab in the treatment of DME. Second, macular oedema was evaluated by HRT II before and after bevacizumab injection, and reduction in oedema map values was demonstrated as the effectiveness of a

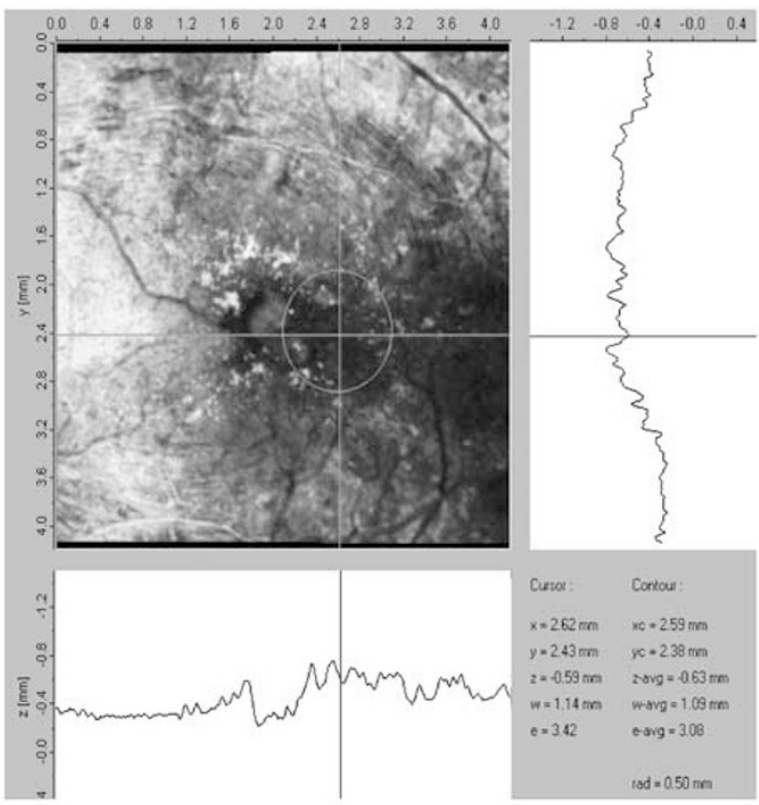

b

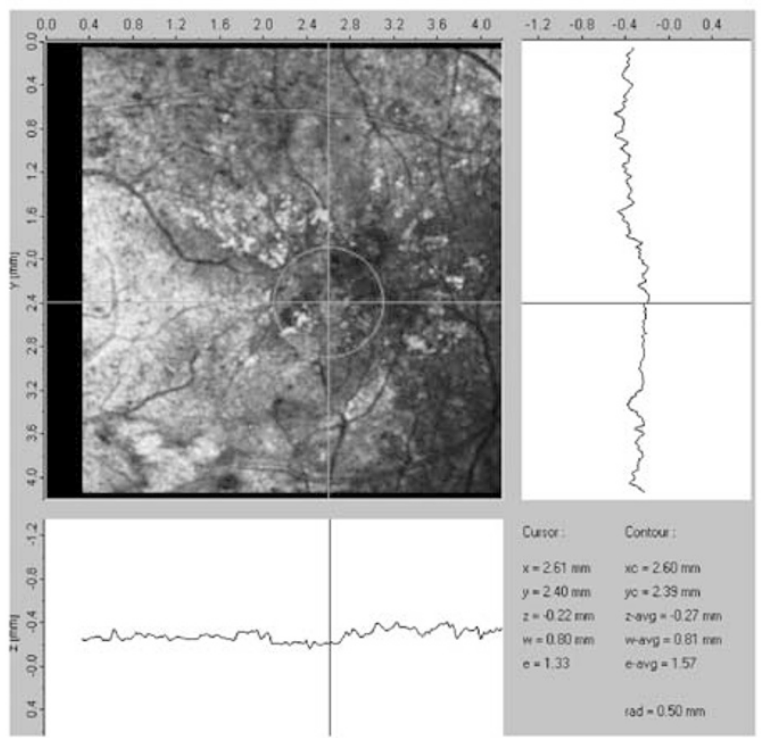

Figure 2 Note the decrease in EM values. (a) Before treatment (b) 6 weeks after intravitreal bevacizumab injection.

bevacizumab application. This study has several limitations. First, the follow-up time was relatively short, but visual and anatomical responses were apparent during the follow-up time. Second, there is no control group in this study, but it can be argued that the enrolled eyes serve as their own controls because the pre-and posttreatment VAs and oedema map values of the same patients were compared. Third, VA was measured on a Snellen chart, as opposed to the more standardized and accepted ETDRS chart. However, all eyes were tested with the same correction throughout the follow-up period.

In conclusion, this study demonstrated that intravitreal bevacizumab application is an effective approach with 
promising results for the primary treatment of DME. Intravitreal bevacizumab provides significant resolution of macular oedema and improvement in VA. However, further studies are needed to obtain the long-term results of such application.

\section{References}

1 Pelzek C, Lim JI. Diabetic macular edema: review and update. Ophthalmol Clin North Am 2002; 15: 555-563.

2 Antcliff RJ, Marshall J. The pathogenesis of edema in diabetic maculopathy. Semin Ophthalmol 1999; 14: 223-232.

3 Ciulla TA, Amador AG, Zinman B. Diabetic retinopathy and diabetic macular edema: pathophysiology, screening, and novel therapies. Diabetes Care 2003; 26: 2653-2664.

4 Ozkiris A, Evereklioglu C, Oner A, Erkilic K. Pattern electroretinogram for monitoring the efficacy of intravitreal triamcinolone injection in diabetic macular edema. Doc Ophthalmol 2004; 109: 139-145.

5 Ozkiris A, Evereklioglu C, Erkilic K, Tamcelik N, Mirza E. Intravitreal triamcinolone acetonide injection as primary treatment for diabetic macular edema. Eur J Ophthalmol 2004; 14: 543-549.

6 Ozkiris A, Erkilic K, Koc A, Mistik S. Effect of atorvastatin on ocular blood flow velocities in patients with diabetic retinopathy. Br J Ophthalmol 2007; 91: 69-73.

7 Early Treatment Diabetic Retinopathy Study Research Group. Treatment techniques and clinical guidelines for photocoagulation of diabetic macular edema. Early Treatment Diabetic Retinopathy Study Report Number 2. Ophthalmology 1987; 94: 761-774.

8 Jonas JB, Kamppeter BA, Harder B, Vossmerbaeumer U, Sauder G, Spandau UH. Intravitreal triamcinolone acetonide for diabetic macular edema: a prospective, randomized study. J Ocul Pharmacol Ther 2006; 22: 200-207.

9 Martidis A, Duker JS, Greenberg PB, Rogers AH, Puliafito $\mathrm{CA}$, Reichel $\mathrm{E}$ et al. Intravitreal triamcinolone for refractory diabetic macular edema. Ophthalmology 2002; 109: 920-927.

10 Otani T, Kishi S. A controlled study of vitrectomy for diabetic macular edema. Am J Ophthalmol 2002; 134: 214-229.

11 Yamamoto T, Akabane N, Takeuchi S. Vitrectomy for diabetic macular edema: the role of posterior vitreous detachment and epimacular membrane. Am J Ophthalmol 2001; 132: 369-377.

12 Aiello LP. The potential role of PKC beta in diabetic retinopathy and macular edema. Surv Ophthalmol 2002; 47: S263-S269.

13 Nguyen QD, Tatlipinar S, Shah SM, Haller JA, Quinlan E, Sung J et al. Vascular endothelial growth factor is a critical stimulus for diabetic macular edema. Am J Ophthalmol 2006; 142: 961-969.

14 Presta LG, Chen H, O'Connor SJ, Chisholm V, Meng YG, Krummen $\mathrm{L}$ et al. Humanization of an anti-vascular endothelial growth factor monoclonal antibody for the therapy of solid tumors and other disorders. Cancer Res 1997; 57: 4593-4599.

15 Chen Y, Wiesmann C, Fuh G, Li B, Christinger HW, McKay $P$ et al. Selection and analysis of an optimized anti-VEGF antibody: crystal structure of an affinity-matured Fab in complex with antigen. J Mol Biol 1999; 293: 865-881.

16 Yoganathan P, Deramo VA, Lai JC, Tibrewala RK, Fastenberg DM. Visual improvement following intravitreal bevacizumab (Avastin) in exudative age-related macular degeneration. Retina 2006; 26: 994-998.

17 Iliev ME, Domig D, Wolf-Schnurrbursch U, Wolf S, Sarra GM. Intravitreal bevacizumab (Avastin) in the treatment of neovascular glaucoma. Am J Ophthalmol 2006; 142: 1054-1056.

18 Oshima Y, Sakaguchi H, Gomi F, Tano Y. Regression of iris neovascularization after intravitreal injection of bevacizumab in patients with proliferative diabetic retinopathy. Am J Ophthalmol 2006; 142: 155-158.

19 Jorge R, Costa RA, Calucci D, Cintra LP, Scott IU. Intravitreal bevacizumab (Avastin) for persistent new vessels in diabetic retinopathy (IBEPE study). Retina 2006; 26: 1006-1013.

20 Haritoglou C, Kook D, Neubauer A, Wolf A, Priglinger S, Strauss $\mathrm{R}$ et al. Intravitreal bevacizumab (Avastin) therapy for persistent diffuse diabetic macular edema. Retina 2006; 26: 999-1005.

21 Holladay JT. Proper method for calculating average visual acuity. J Refr Surg 1997; 13: 388-391.

22 Zambarakji HJ, Evans JE, Amoaku WM, Vernon SA. Reproducibility of volumetric measurements of normal maculae with the Heidelberg retina tomograph. $\mathrm{Br}$ J Ophthalmol 1998; 82: 884-891.

23 Ozkiris A, Erkilic K. Complications of intravitreal injection of triamcinolone acetonide. Can J Ophthalmol 2005; 40: 63-68.

24 Jonas JB, Kreissig I, Degenring RF. Retinal complications of intravitreal injections of triamcinolone acetonide. Graefes Arch Clin Exp Ophthalmol 2004; 242: 184-185.

25 Cunningham Jr ET, Adamis AP, Altaweel M, Aiello LP, Bressler NM, D'Amico DJ et al. Macugen Diabetic Retinopathy Study Group. A phase II randomized double-masked trial of pegaptanib, an anti-vascular endothelial growth factor aptamer, for diabetic macular edema. Ophthalmology 2005; 112: 1747-1757.

26 Costa RA, Jorge R, Calucci D, Melo Jr LA, Cardillo JA, Scott IU. Intravitreal bevacizumab (avastin) for central and hemicentral retinal vein occlusions: IBeVO study. Retina 2007; 27: 141-149.

27 Friedlander SM, Welch RM. Vanishing disc neovascularization following intravitreal bevacizumab (avastin) injection. Arch Ophthalmol 2006; 124: 1365.

28 Feiner L, Barr EE, Shui YB, Holekamp NM, Brantley Jr MA. Safety of intravitreal injection of bevacizumab in rabbit eyes. Retina 2006; 26: 882-888.

29 Ziemssen F, Grisanti S, Bartz-Schmidt KU. The international intravitreal bevacizumab safety survey. $\mathrm{Br}$ J Ophthalmol 2006; 90: 1440-1441.

30 Pai SA, Shetty R, Vijayan PB, Venkatasubramaniam G, Yadav NK, Shetty BK et al. Clinical, anatomic, and electrophysiologic evaluation following intravitreal bevacizumab for macular edema in retinal vein occlusion. Am J Ophthalmol 2007; 143: 601-606.

31 Augustin AJ, Puls S, Offermann I. Triple therapy for choroidal neovascularization due to age-related macular degeneration: verteporfin PDT, bevacizumab, and dexamethasone. Retina 2007; 27: 133-140.

32 Ziemssen F, Deuter CM, Stuebiger N, Zierhut M. Weak transient response of chronic uveitic macular edema to intravitreal bevacizumab (Avastin). Graefes Arch Clin Exp Ophthalmol 2007; 245(6): 917-918.

33 Mason III JO, Albert Jr MA, Vail R. Intravitreal bevacizumab (Avastin) for refractory pseudophakic cystoid macular edema. Retina 2006; 26: 356-357. 